\title{
MARKOV CHAINS WITH QUASITOEPLITZ TRANSITION MATRIX: APPLICATIONS ${ }^{\star}$
}

\author{
A. M. Dukhovny \\ Department of Mathematics \\ San Francisco State University \\ San Francisco, CA 94132
}

\begin{abstract}
Application problems are investigated for the Markov chains with quasitoeplitz transition matrix. Generating functions of transient and steady state probabilities, first zero hitting probabilities and mean times are found for various particular cases, corresponding to some known patterns of feedback ("warm-up," "switch at threshold," etc.). Level-depending dams and queue-depending queueing systems of both $M / G / 1$ and GI/M/1 types with arbitrary random sizes of arriving and departing groups are studied.
\end{abstract}

Key words: Markov chains, quasitoeplitz matrix, dams, queueing systems, feedback, arbitrary group size.

AMS subject classification: $60 \mathrm{~J}, 60 \mathrm{~K} 25$.

\section{INTRODUCTION}

In this paper we investigate the application problems for the general results of our preceding papers [1] and [2] on the Markov chains with quasitoeplitz transition matrix, elements of which depend on the difference of indices except for the first column and some first rows.

We consider here some particular cases of this structure with various types of relationships between the toeplitz part of the transition matrix and its first nontoeplitz rows. These relationships correspond to some types of state-dependence known in literature:

- $\quad$ "warm-up," with one row in the nontoeplitz part;

- $\quad$ "Bailey type," with equal first rows;

- $\quad$ "switch at a threshold," with the first rows of another toeplitz structure.

We refer, for example, to Abolnikov and Dukhovny [3, 4] and Neuts [5] for results on transient and steady state probabilities and the ergodicity criterion in the case where toeplitz rows have left or right zero parts.

Here we proceed from the general results of [1] and [2] to specify formulas for the generating functions (GF's) of the transient and steady state probabilities, first zero hitting (FZH) probabilities and mean times, and the ergodicity criterion for all mentioned patterns of state-dependence, with no significant restriction put on the toeplitz part.

\footnotetext{
* Received: September 1989, Revised: March 1990.
} 
We introduce here the model of a dam with additional irregular loss ("vaporization"), being significant only over some dam level, and we treat it as a particular case of Markov chains with quasitoeplitz transition matrix with a switch at a threshold.

Bulk queueing systems $\mathrm{M} / \mathrm{G} / 1$ and $\mathrm{GL} / \mathrm{M} / 1$ are considered with no restriction put on the size distributions of arriving and departing groups. They are also treated as a particular case of the general quasitoeplitz model.

\section{GENERAL RESULTS TABLE}

To make this paper self-contained, we list here assumptions, denotations and results of [1] and [2].

Let Markov chain $\left\{\xi_{k}\right\}$ have a quasitoeplitz transition matrix $A=\left(a_{i j}\right)$, so that the GF's of its elements,

$$
\sum_{j=0}^{\infty} a_{i j} z^{j}=A_{i}(z)=T^{0} z^{i} H(z), \quad i>n>1,
$$

where operators $T^{+}, T^{-}$, and $T^{0}$ are defined as follows.

for any Laurent series $K(z) ; H(z)=\sum_{-\infty}^{\infty} h j z^{j}$.

$$
\begin{aligned}
T^{+} K(z) & =\sum_{j=0}^{\infty} k_{j} z^{j}, \quad T-K(z)=\sum_{j=-\infty}^{-1} k_{j} z^{j}, \\
T^{0} K(z) & =\sum_{j=0}^{\infty} k_{j} z^{j}+\sum_{j=-\infty}^{-1} k_{j}
\end{aligned}
$$

(See section 3 for $n=0$.)

Natural properties (see [1]) emerge - conditions of descent and ascent (enabling the chain to descend to zero from any other state, to stay at zero, to ascend from zero to above $n)$ and the existence of averages $\left(\sum_{j=0}^{\infty} j a_{i j}<\infty\right.$, for all $i$.)

$$
H^{\prime}(1)<0 \text {, }
$$

so $\left\{\xi_{k}\right\}$ is ergodic and, as it was proved in [1],

$$
\text { Ind }_{\Gamma}[1-H(z)] z e(z)=0,
$$

where $e(z)=\sum_{1}^{\infty} z^{-i} ; \Gamma:|z|=1, \Gamma^{+}:|z|<1, \Gamma:|z|>1$.

Therefore, functions

$$
\begin{gathered}
R^{ \pm}(x, z)=\exp \left\{-T^{ \pm} \ln [1-x H(z)]\right\}, x \in(0,1), \\
R^{ \pm}(z)=\exp \left\{-T^{ \pm} \ln z e(z)[1-H(z)]\right\}
\end{gathered}
$$

and their reciprocals are analytical in $\Gamma^{+}$and $\Gamma^{2}$, respectively, and continuous on $\Gamma$.

The GF $W(x, z)=\sum_{k=0}^{\infty} \sum_{j=0}^{\infty} p_{j}^{k} z j x^{k}$ of the transient state probabilities is determined by 


$$
W(x, z)=\sum_{i=0}^{n-1} U_{i}(x) \cdot\left[z^{i}+x z^{n} R^{+}(x, z) T^{+} z^{-n} A_{i}(z) R^{-}(x, z)\right]
$$

where $U_{0}(x), U_{1}(x), \ldots, U_{n-1}(x)$ form the only solution of the system of linear equations

$$
\sum_{i=0}^{n} U_{j}(x) D^{j} T^{0}\left[z^{i}-x A_{i}(z)\right] R^{-}(\mathrm{x}, \mathrm{z})=\delta_{j}^{0} R^{-}(x, 1), j=0, \ldots, n-1 .
$$

By definition, $D_{j} K(z)=k_{j}$ for any Laurent series $K(z)$.

The GF $P(z)=\sum_{0}^{\infty} p_{j} z^{j}$ of the steady state probabilities is

$$
P(z)=\sum_{i=0}^{n-1} p_{i}\left[z^{i}+z^{n} R^{+}(z) T^{+} z^{1-n} e(z) A_{i}(z) R^{-}(z)\right]
$$

where $p_{0}, p_{1}, \ldots, p_{n-1}$, and $c$ form the only solution of the system of linear equations:

$$
\sum_{i=0}^{n} p_{i} D^{j} T^{0} z e(z)\left[z^{i}-A_{i}(z)\right] R^{-}(z)=c \delta_{j}^{0}, j=0,1, \ldots, n-1
$$

(where $\delta_{j}^{0}$ stands for Kronecker's symbol),

$$
c R^{+}(1)+\sum_{0}^{n} p_{i}\left\{1-H^{\prime}(1)^{-1}\left[A_{i}^{\prime}(1)-i\right]\right\}=1 .
$$

Let $q_{i}^{k}, i>0, k>0$, be the probability of FZH in $k$ steps from $i, q_{i}^{0}=-1$, $q_{i}(x)=\sum_{k=0}^{\infty} q_{i}^{k} x^{k} ; Q(x, z)=\sum_{1}^{\infty} q_{i}(x) z^{i}$. Then

$$
Q(x, z)=z^{1-n} R^{-}(x, z)\left[(x-1) R^{+}(x, 1) e(z)+\sum_{i=1}^{n} q_{i}(x) T^{+} z^{n-1-i} R^{-}(x, z)^{-1}\right],
$$

and $q_{0}(x), q_{1}(x), \ldots, q_{n-1}(x)$ form the only solution of the system of linear equations $(i=0,1, \ldots, n-1)$ :

$$
\begin{gathered}
q_{i}(x)-x \sum_{j=1}^{n} q_{j}(x) D^{n-1} A_{i}(z) R^{-}(x, z) T^{+} z^{\mathrm{n}-1-j} \cdot R^{-}(x, z)^{-1} \\
=(x-1)\left[1+x R^{+}(x, 1) D^{n-1} A_{i}(z) e(z) R^{-}(x, z)\right] .
\end{gathered}
$$

When $i=0$ (12) is a formula for the $q_{0}(x)=\sum_{k=1}^{\infty} q_{0}^{k} x^{k}-1$ where the $q_{0}^{k}$ 's are the probabilities of the first return to 0 .

The GF $\mu(z)$ of FZH mean times $\mu_{i}=\sum_{k=1}^{\infty} k q_{i}^{k}$ is

$$
\mu(z)=z^{2-n} e(z) R^{-}(z)\left[R^{+}(1) e(z)+\sum_{i=1}^{n} \mu_{i} T^{+} z^{n-i-2}(z-1) R^{-}(z)^{-1},\right.
$$

where $\mu_{0}, \mu_{1}, \ldots, \mu_{n-1}$ form the only solution of the system of linear equations $(i=0,1, \ldots, n-1)$, 


$$
\begin{gathered}
\mu_{i}-\sum_{j=1}^{n} \mu_{j}^{-1} D^{n-2} A_{i}(z) R^{-}(z) e(z) T^{+} z^{n-j-2}(z-1) R^{-}(z)^{-1} \\
=1+R^{+}(1) D^{n-2} A_{i}(z) e^{2}(z) R^{-}(z) .
\end{gathered}
$$

When $i=0$ (14) provides a formula for $\mu_{0}$, which is the mean time of the first return to zero.

\section{SIMPLE PARTICULAR CASES}

In this section we consider some simple particular cases of the general model.

a) $n=0$-- homogeneous random walk with absorption in zero, no statedependence.

Combining corresponding results of [1] and [2] we have

$W(x, z)=R^{-}(x, 1) R^{+}(x, z)=(1-x)^{-1} R^{+}(x, 1)^{-1} R^{+}(x, z)$,

$P(z)=R^{+}(1)^{-1} R^{+}(z)$,

$Q(x, z)=(x-1) e(z) R^{+}(x, 1) R^{-}(x, z)$,

$$
q_{0}(x)=(x-1) R^{+}(x, 1) R^{+}(x, 0)^{-1} \text {, }
$$

$\mu(z)=z e^{2}(z) R^{+}(1) R^{-}(z)$,

$\mu_{0}=R^{+}(1) R^{+}(0)^{-1}$.

Note that it follows from (15) and (16) that

$$
\begin{gathered}
U_{0}(x)=(1-x)^{-1} R^{+}(x, 1)^{-1} R^{+}(x, 0), \\
p_{0}=R^{+}(1)^{-1} R^{+}(0), \\
-U_{0}(x) q_{0}(x)=p_{0} \mu_{0}=1
\end{gathered}
$$

in accordance with the renewal processes theory.

b) $n=1$ - "warm-up."

To provide the conditions of descent and ascent we only need that $0<a_{00}<1$. All the GF's still may be derived explicitly from formulas (6) through (14):

(22) $W(x, z)=U_{0}(x)\left[1+x z R^{+}(x, z) T^{+} z^{-1} A_{0}(z) R^{-}(x, z)\right]$;

(23) $\quad U_{0}(x)^{-1}=(1-x)\left[1+x R^{+}(x, 1) D^{\circ} A_{0}(z) e(z) R^{-}(x, z)\right]$;

(24) $P(z)=p_{0}\left[1+z R^{+}(z) T^{+} e(z) A_{0}(z) R^{-}(x)\right]$;

(25) $\quad p_{0}^{-1}=1+R^{+}(1) D^{\circ} A_{0}(z) e^{2}(z) R^{-}(z)$.

$Q(x, z)$ and $\mu(z)$ are given by (17) and (19), so

$q_{0}(x)=(x-1)\left[1+x R^{+}(x, 1) D^{\circ} A_{0}(z) e(z) R^{-}(x, z)\right]$,

(27) $\mu_{0}=1+R^{+}(1) D^{\circ} A_{0}(z) e^{2}(z) R^{-}(z)$. 
To obtain formulas for $U_{0}(x)$ and $p_{0}$, we have used normalizing equalities $W(x, 1)=(1-x)^{-1}$ and $p(1)=1$, respectively, and one simple identity for functions with absolutely summable Laurent coefficients: if $a(z)=\sum_{-\infty}^{\infty} a_{j} z^{j} \in L^{1}$, then

$$
\left.\left[T^{+} a(z)\right]\right|_{z=1}=\sum_{j=0}^{\infty} a_{j}=D^{\circ} z a(z) e(z) .
$$

Hence, equality (21) also holds here.

In some applications, for example queueing systems with waiting server, the system's being empty may cause an additional input with GF, say, $b(z)=\sum_{0}^{\infty} b_{j} z^{j}$, so $A_{0}(z)=T^{0} b(z) H(z)$, and we can use the formulas above.

Let $b(z)=z$, then

$$
\begin{gathered}
W(x, z)=(1-x)^{-1} R^{+}(x, 1)^{-1}\left[1-z+z R^{+}(x, z)\right], \\
P(z)=R^{+}(1)^{-1}\left[1-z+z R^{+}(z)\right] .
\end{gathered}
$$

The latter is a generalization of the Pollyatchek-Khinchin formula, that follows from here when $H(z)=K(z) z^{-1}=\sum_{0}^{\infty} k_{i} z^{i-1}$ so $R^{+}(z)=(z-1)[z-K(z)]^{-1}$.

\section{PARTICULAR CASES WITH $n>1$}

c) For example, $A_{i}(z)=A(z), i=0,1, \ldots, n-1$, is the generalization of the case that takes place in Bailey's bulk queue model.

In [5] Bailey's model is treated by a method, depending on the server's capacity being finite, that is, there is some $m$ such that, for all $j .<-m, h_{j}=0$. In our study this restriction is not necessary. The conditions of descent and ascent are guaranteed by $0<a_{00}<1$. Denote

$$
\hat{W}=\sum_{0}^{n} U_{i}(x) z^{i} ; \hat{P}(z)=\sum_{0}^{n} p_{i}^{1} z^{i} ; S(x)=\hat{W}(x, 1) ; S=\hat{P}(1) .
$$

Theorem 1. The GF of the transient and steady state probabilities, the FZH probabilities and the mean times for the Markov chain with quasitoeplitz transition matrix of "Bailey type" when $A_{i}(z)=A(z), i=0,1, \ldots, n-1$, are given by the formulas:

(31) $W(x, z)=1+x S(x)\left[T^{0} z^{n} R^{-}(x, z)^{-1} T^{-} z^{-n} A(z) R^{-}(x, z)+z^{n} R^{+}(x, z) T^{+} z^{-n} A(z) R^{-}(x, z)\right]$,

$$
\begin{gathered}
S(x)^{-1}=(1-x) D^{n} A(z) R^{-}(x, z)\left[e\left(z^{-1}\right) R^{-}(x, 1)^{-1}+z e(z) R^{+}(x, 1)\right] \\
P(z)=S\left[T^{0} z^{n-1}(z-1) R^{-}(z)^{-1} T^{-} z^{1-n} e(z) A(z) R^{-}(z)+z^{n} R^{+}(z) T^{+} z^{1-n} e(z) A(z) R^{-}(z)\right], \\
S^{-1}=1+R^{+}(1) D^{n-2} A(z) e^{2}(z) R^{-}(z), \\
Q(x, z)=z^{1-n} R^{-}(x, z)\left[(x-1) R^{+}(x, 1) e(z)+q_{0}(x) T^{+} z^{n-1} e(z) R^{-}(x, z)^{-1}\right] \\
q_{i}(x)=\left[1+x R^{+}(x, 1) D^{n-1} A(z) e(z) R^{-}(x, z)\right]: \\
{\left[1-x D^{n-1} A(z) R^{-}(x, z) T^{+} z^{n-1} e(z) R^{-}(x, z)^{-1}\right], i<n,}
\end{gathered}
$$




$$
\begin{aligned}
\mu(z)= & z^{2-n} e(z) R^{-}(z)\left[R^{+}(1) e(z)+\mu_{0} T^{+} z^{n-2} R^{-}(z)^{-1}\right] \\
\mu_{i}= & {\left[1+R^{+}(1) D^{n-2} A(z) e^{2}(z) R^{-}(z)\right]: } \\
& {\left[1-D^{n-2} A(z) R^{-}(z) e(z) T^{+} z^{n-2} R^{-}(z)^{-1}\right], \quad i<n . }
\end{aligned}
$$

Proof. When $A_{i}(z)=A(z)$, for all $i<n$, then (7) becomes $\operatorname{DiT}^{0} \hat{W}(x, z) R^{-}(x, z)=\delta_{j}^{0} R^{-}(x, 1)+x S(x) \operatorname{DjT}^{0} A(z) R^{-}(x, z), j<n$. Due to the definitions of $\hat{W}(x, z)$ and $T^{-}$this yields

$$
T^{-} z^{-n} T^{0} \hat{W}(x, z) R^{-}(x, z)=R^{-}(x, 1) z^{-n}+x S(x) T^{-} z^{-n} T A(z) R^{-}(x, z) .
$$

Two obvious identities directly follow from the definitions of operators $T^{+}, T^{-}, T^{0}$. Let $f(z)$ and $a^{-}(\mathrm{z})$ both belong to $L^{1}$ and $a^{-}(z)$ be analytical in $\Gamma^{2}$. Then

$$
T^{0} a^{-}(z)=a^{-(1)}
$$

and hence

$$
T^{0} z^{n} a^{-}(z) T^{-} z^{-n} T^{0} f(z)=T^{0} z^{n} a^{-}(z) T^{-} z^{-n} f(z),
$$

as $f(z)-T^{0} f(z)$ is analytical in $\Gamma^{2}$ and is 0 when $z=1$.

Now we multiply both sides of (39) by $z^{n} R^{-}(x, z)^{-1}$, apply $T^{0}$, use these identities and obtain

(40) $T^{0} z^{n} R^{-}(x, z)^{-1} T^{-} z^{-n} \hat{W}(x, z) \cdot R^{-}(x, z)=1+\mathrm{xS}(\mathrm{x}) T^{0} z^{n} R^{-}(x, z)^{-1} T^{-} z^{-n} A(z) R^{-}(x, z)$.

$T^{-}$does not change $z^{-n} \hat{W}(x, z) R^{-}(x, z)$ as it is analytical in $\Gamma^{\text {, }}$, so the left side of (40) is $\hat{W}(x, z)$. Using (40) in (6), we obtain (30).

Using (31) in the equality $W(x, 1)=(1-x)^{-1}$,we obtain (32) with the help of (28) and obvious identities:

$$
\begin{aligned}
& {\left.\left[T^{0} f(z)\right]\right|_{z}=1=f(1),} \\
& D^{0} z^{n} f(z)=D^{-n} f(z) .
\end{aligned}
$$

(33) and (34) are obtained by the same method.

Considering FZH problems we can see that for the Bailey type transition matrix, (12) looks like

$$
\begin{aligned}
q_{i}= & x \sum_{j=1}^{n-1} q_{j}(z) D^{n-1} A(z) R^{-}(x, z) T^{+} z^{n-1-j} R^{-}(x, z)^{-1} \\
& +(x-1)\left[1+x R^{+}(x, z) D^{n-1} A(z) e(z) R^{-}(x, z)\right] .
\end{aligned}
$$

Since the right side of this doesn't depend on $i$, then all $q_{i}(x), i<n$, are equal, so (35) and (36) follow immediately, as

$$
\sum_{j=1}^{n-1} z^{n-1-j}=\left(z^{n}-1\right) e(z), T^{+} e(z) R^{-}(x, z)^{-1}=0 .
$$

Formulas (38) and (39) are obtained in the same manner. 
b) $\quad A_{i}(z)=z^{i} \hat{H}(z), i<n,-$ a switch at a threshold $n$. To provide the conditions of descent and ascent we assume that the Laurent expansion of $\hat{H}(z)$ contains positive and negative powers of $z$ with nonzero coefficients.

Formulas (6) to (14) give all the GF's that we need if we replace $A_{i}(z)$ by $z^{i} \hat{H}(z), i<n$.

\section{CALCULATION PROBLEMS}

The efficiency of calculations based on the aforementioned formulas strongly depends on the possibility of calculating effectively the functions $R^{ \pm}(x, z)$ and $R^{ \pm}(z)$ and the effect of the operators $D^{i}, T^{+}, T^{-}$, and $T^{0}$.

With all the models in applications being approximate, one can always design a model in which $H(z)$ is approximated with a function that is meromorphically extensible to either $\Gamma^{+}$or $\Gamma^{*}$. In fact, it is sufficient to replace either the negative-indexes part of $\left\{h_{j}\right\}$ (for $\Gamma^{+}$) or the positive-indexes part of it (for $\Gamma^{2}$ ) with respective parts of some suitable function, or even simply to truncate these parts at some level. It can be done with all necessary precision. (To retain the ergodicity criterion and provide the necessary proximity between $R^{ \pm}(z)$ and $\hat{R}^{ \pm}(z)$, corresponding to approximate $\hat{H}(z)$, we must estimate such approximation in terms of $\sum_{-\infty}^{\infty}\left|j h_{j}-j \hat{h}_{j}\right|$.)

It must also be mentioned that this analytical property of $H(z)$ arises in applications in the most natural manner. In fact, no other cases were in study to date.

Lemma 1. Let the ergodicity criterion (2) be true and $\left\{h_{j}\right\}$ be an aperiodic sequence.

1) If $H(z)$ is meromorphically extensible to $\Gamma^{+}, \varepsilon_{1}, \varepsilon_{2}, \ldots, \varepsilon_{m}$ being its poles in $\Gamma^{+}$(some of them may be equal), then $1-x H(z)$ and $z[1-H(z)] e(z)$ have exactly $m$ roots in $\Gamma^{+}$, $a_{i}(x)$ and $a_{i}$, respectively, and

$$
\begin{array}{ll}
R^{-}(x, z)=\prod_{i=1}^{m}\left(z-\varepsilon_{i}\right)\left(z-a_{i}(x)\right)^{-1}, & R^{+}(x, z)^{-1}=[1-x H(z)] R^{-}(x, z), \\
R^{-}(z)=\prod_{i=1}^{m}\left(z-\varepsilon_{i}\right)\left(z-a_{i}\right)^{-1}, & R^{+}(z)^{-1}=e(z)[1-H(z)] R^{-}(z) .
\end{array}
$$

2) If $H(z)$ is meromorphically extensible to $\Gamma^{2}, \varepsilon_{1}, \varepsilon_{2}, \ldots, \varepsilon_{m}$ being its poles there (some of them may be equal), then $1-x H(z)$ and $1-H(z)$ have exactly m roots in $\Gamma^{2}, a_{i}(x)$ and $a_{i}$, respectively, and

$$
R^{+}(x, z)=k(x) \prod_{i=1}^{m}\left(z-\varepsilon_{i}\right)\left(z-a_{i}(x)\right)^{-1}, \quad R^{-}(x, z)^{-1}=[1-x H(z)] R^{+}(x, z),
$$




$$
R^{+}(z)=k \prod_{i=1}^{m}\left(z-\varepsilon_{i}\right)\left(z-\alpha_{i}\right)^{-1}, \quad \quad R^{-}(z)^{-1}=e(z)[1-H(z)] R^{+}(z) .
$$

where $k(x)$ and $k$ are some normalizing coefficients.

Proof. It was proved in [1] that Ind $\Gamma$ of the functions $1-x H(z)$ and $z[1-H(z)] e(z)$ is zero, provided $x \in(0,1)$ or $H^{\prime}(1)<0$, respectively. For the functions meromorphic in $\Gamma^{+}$or $\Gamma^{-}$, Ind $_{\Gamma}$ is the difference between the numbers of roots and poles in $\Gamma^{+}\left(\Gamma^{-}\right)$, so these numbers are equal.

It is known (see, for example, Gahov [6]) that if $f(z)$ is continuous on $\Gamma$ and Ind $f(z)=0$, then $f^{ \pm}(z)=\exp \left\{-T^{ \pm} \ln f(z)\right\}$ are the only functions analytical and nonzero in $\Gamma^{+}$and $\Gamma^{2}$ respectively, such that the factorization identity holds on $\Gamma$ :

$$
f(z)^{-1}=f^{+}(z) f^{-}(z) ; f^{-}(\infty)=1 .
$$

Let us prove, for example, (41). With $\varepsilon_{i}$ and $\alpha_{i}(x)$ belonging to $\Gamma^{+}, R^{-}(x, z)$ given is obviously analytical and nonzero in $\Gamma^{2}$, and $R^{-}(x, \infty)=1$. Due to the definitions of $\varepsilon_{i}$ and $\alpha_{i}(x), R^{+}(x, z)$ given by $(41)$ has no roots or poles in $\Gamma^{+}$, so it is analytically extensible to $\Gamma^{+}$. Equation (41) also ensures that the factorization identity holds for $1-x H(z)$.

The other parts of Lemma 1 have a similar proof, but in case 2) we introduce normalizing coefficients $k(x)$ and $k$ to ensure $R^{-}(x, \infty)=1$ and $R^{-}(\infty)=1$.

Given the aforementioned analytical properties of $H(z)$, the following lemma makes it possible for us to calculate the effect of operators $T^{+}$and $T^{-}$.

Denote $L_{m-1}(z)$ as the Lagrange polynomial, interpolating the function $f(z)$ values at some points $\varepsilon_{1}, \ldots, \varepsilon_{m}$.

\section{Lemma 2.}

1) Let $f(z)$ be analytical in $\Gamma^{+}, \varepsilon_{i} \in \Gamma^{+}, i=1, \ldots, m$.

$$
\begin{gathered}
T^{-} f(z) \prod_{i=1}^{m}\left(z-\varepsilon_{i}\right)^{-1}=L_{m-1}(z) \prod_{i=1}^{m}\left(z-\varepsilon_{i}\right)^{-1}, \\
T^{+} f(z) \prod_{i=1}^{m}\left(z-\varepsilon_{i}\right)^{-1}=\left[f(z)-L_{m-1}(z)\right] \prod_{i=1}^{m}\left(z-\varepsilon_{i}\right)^{-1} .
\end{gathered}
$$

2) Let $f(z)$ be analytical in $\Gamma^{+}, \varepsilon_{i} \in \Gamma^{-}, i=1, \ldots, m$.

$$
\begin{gathered}
T^{-} f(z) \prod_{i=1}^{m}\left(z-\varepsilon_{i}\right)^{-1}=\left[f(z)-L_{m-1}(z)\right] \prod_{i=1}^{m}\left(z-\varepsilon_{i}\right)^{-1}-\varepsilon . \\
T^{+} f(z) \prod_{i=1}^{m}\left(z-\varepsilon_{i}\right)^{-1}=L_{m-1}(z) \prod_{i=1}^{m}\left(z-\varepsilon_{i}\right)^{-1}+\varepsilon,
\end{gathered}
$$

where

$$
\varepsilon=\lim _{z \rightarrow \infty} f(z) \prod_{i=1}^{m}\left(z-\varepsilon_{i}\right)^{-1}
$$


Proof. We must prove that formulas (45) to (48) provide functions $T^{ \pm} f(z)$ that are analytical in $\Gamma^{+}$and $\Gamma^{2}$, respectively, which readily follows from the definition of $L_{m-1}(z)$; these formulas also insure that, on $\Gamma, f(z)=T^{+} f(z)+T^{-} f(z)$.

The definition of $T$ implies that $\lim _{z \rightarrow \infty} T^{-} f(z)=0$, as it contains only powers of $z$ with negative exponents. In case 1) it is guaranteed by the fact that

$$
\lim _{z \rightarrow \infty} L_{m-1}(z) \prod_{i=1}^{m}\left(z-\varepsilon_{i}\right)^{-1}=0
$$

because the greatest power of $\mathrm{z}$ in $L_{m-1}(z)$ is $m-1$. In case 2$)$ we introduce $\varepsilon$ to provide the same limit property.

\section{DAMS WITH A LIMTTED LEVEL DEPENDENCE}

A classical model of a dam in discrete time with discretized content and infinite capacity can be described by the Markov chain $\left\{\xi_{k}\right\}$ such that

$$
\xi_{k+1}=\max \left(0, \xi_{k}+\alpha-\beta\right)
$$

where $\alpha$ and $\beta$ are the inflow and the outflow over the control time period $\left(t_{k}, t_{k+1}\right)$. As a rule $\alpha$ and $\beta$ are considered independent random variables with their distributions not depending on $k$. We consider here the model of a dam with a limited level dependence, where $\alpha$ and $\beta$ distributions over the interval $\left(t_{k}, t_{k+1}\right)$ depend on the value of $\xi_{k}$ in such a way that beginning from some $n$ their distributions don't change.

With this assumption one can easily see that $\left\{\xi_{k}\right\}$ has a quasitoeplitz transition matrix and

$$
\begin{gathered}
A_{i}(z)=T^{0} z^{i} a_{i}(z) b_{i}\left(z^{-1}\right), \quad i<n, \\
A_{i}(z)=T^{0} z^{i} H(z), \quad i<n ; \quad \mathrm{H}(\mathrm{z})=a_{n}(z) b_{n}\left(z^{-1}\right),
\end{gathered}
$$

where $a_{i}(z)=E\left\{z^{\alpha} ; \xi=i\right\}, b_{i}(z)=E\left\{z^{\beta} ; \xi=i\right\}$.

The natural properties of the transition matrix are guaranteed by the trivial assumptions:

— for each $i, \beta$ may exceed $\alpha$ (for descent);

- for each $i<n, \alpha$ may exceed $\beta$ (for ascent);

- for each $i, \alpha$ and $\beta$ have finite means.

The ergodicity criterion (2) looks like

$$
a_{n}^{\prime}(1)<b_{n}^{\prime}(1) \text {. }
$$

Thus we may apply all the formulas (4) through (14) in this case with respect to (51) and (52). No significant restrictions must be put on the sizes of $\alpha$ and $\beta$, as they were 
before, so there is room for various complex models, with more features reflecting practical problems.

Let's introduce, for example, the model with "vaporization" that becomes significant at some level $n$. This means that besides the regular release $m$ there is also additional irregular loss $\beta_{v}$. The data available may contain information sufficient to estimate only the order of decline for probabilities of the higher values of $\beta_{v}$, not the upper bound of it. This makes it reasonable to simulate $\beta_{v}$ as not limited, with a GF $b_{v}(z)$ that is a rational function with the prescribed decline of coefficients.

Hence, here we have a switch at the threshold $n$, and

$$
\begin{gathered}
\hat{H}(z)=z^{-m} a(z) \\
\mathrm{H}(\mathrm{z})=z^{-m} b_{v}\left(z^{-1}\right) a(z) .
\end{gathered}
$$

The ergodicity criterion (53) herewith looks like

$$
a^{\prime}(1)<m+b_{v}^{\prime}(1) \text {. }
$$

As $b_{v}\left(z^{-1}\right)$ is a rational function with the poles in $\Gamma^{+}$, we can calculate $R^{ \pm}(x, z)$, $R^{ \pm}(z)$ and the effects of the operators $T^{+}, T^{-}$, and $T^{0}$ with the help of Lemmas 1 and 2 .

\section{QUEUEING SYSTEMS WITH A LIMITED QUEUE LENGTH DEPENDENCE}

In this section we consider the queueing systems of $M / G / 1$ and $G I / M / 1$ types with operating modes depending on queue length at some special control moments.

Namely, let $t_{k}$ be the moments immediately following service completions in $M / G / 1$ or preceding arrivals in $\mathrm{GI} / \mathrm{M} / 1$, and let $\xi_{k}$ be the queue length at these moments. The operating mode of the system results in $\gamma=\alpha-\beta$ - the difference between the numbers of customers admitted to and taken from the waiting line between $t_{k}$ and $t_{k+1}$. Obviously

$$
\xi_{k+1}=\max \left(0, \xi_{k}+\gamma\right) \text {. }
$$

Denote $H_{i}(z)=E\{z \gamma ; \xi=i\}$. We will refer to this dependence on $i$ as limited if $H_{i}(z)=H(z), i \geq n$.

The definition of $t_{k}$ ensures that $\left\{\xi_{k}\right\}$ defined by (56) is a Markov chain for both M/G/1 and GI/M/1 types (imbedded chain); the limited dependence pattern provides a quasitoeplitz structure of its transition matrix.

It is natural for queueing systems that $\gamma$ take on both positive and negative values, no matter how long the queue is, which provides the conditions of descent and ascent. The existence of averages is also the common feature of all the models, so the "natural properties" of the transition matrix are really natural.

Hence, no matter whether we have a $\mathrm{M} / \mathrm{G} / 1$ or GI/M/1 type, or no matter what the group size distributions might be, all the results of sections 2,3 , and 4 are valid here for 
various limited dependence patterns. The system's nature influences only the expressions for $H_{i}(z), i=0,1, \ldots$.

The ergodicity criterion (2) looks like

$$
H_{n}^{\prime}(1)<0 .
$$

Now let us take the $M / G / 1$ system. Usually

$$
H_{i}(z)=b_{i}\left(z^{-1}\right) \int_{0}^{\infty} \exp \left[\lambda_{i} t a_{i}(z)-\lambda_{i} t\right] d G_{i}(t)
$$

where $G_{i}(t)$ is a distribution function of $t_{k+1}-t_{k} ; a_{i}(z)$ and $b_{i}(z)$ are the GF of the arriving group size and server's capacity, respectively, when the queue length at $t_{k}$ is $i$.

In all the previous investigations it was required that the server's capacity be finite. Obviously we don't need it here. New queueing models may be investigated where this capacity may be so large that it is better to treat it as infinite, but with a reasonably adjusted distribution. Take, for example, an airport where neither the number of everyday flights nor their capacities are constant.

Simulating capacity with a suitable rational GF $b_{n}(z)$, we can make use of Lemmas 1 and 2 and perform all the necessary calculations, provided the ergodicity criterion holds. Due to (58) this criterion looks here like

$$
\lambda_{n} a_{n}{ }^{\prime}(1) \int_{0}^{\infty} t d G_{n}(t)<b_{n}{ }^{\prime}(1) .
$$

Now we consider a GI/M/1 system, where usually

$$
H_{i}(z)=a_{i}(z) \int_{0}^{\infty} \exp \left\{v_{i} t b_{i}\left(z^{-1}\right)-v_{i} t\right\} d G_{i}(t)
$$

with the same denotation. We are again able to expand the range of models, to include the cases with indefinite arriving group size, especially with the rational GF.

The ergodicity criterion (2) here looks like

$$
a_{n}^{\prime}(1)<v_{n} b_{n}{ }^{\prime}(1) \int_{0}^{\infty} t d G_{n}(t)
$$

\section{REFERENCES}

[1] Dukhovny, A.M. Markov Chains with Quasitoeplitz Transition Matrix, Journal of Applied Mathematics and Simulation 2(1989):71-82.

[2] Dukhovny, A.M. Markov Chains with Quasitoeplitz Transition Matrix: First Zero Hitting, Journal of Applied Mathematics and Simulation 3(1989), 205-216. 
152 Journal of Applied Mathematics and Stochastic Analysis Volume 3, Number 2, 1990

[3] Abolnikov, L.M. and Dukhovny, A.M., Markov Chains with $\Delta_{m, n}\left(\Delta_{m, n}^{\prime}\right)$ Transition Matrix and Their Applications to the Queueing Systems with Bulk Arrival, Batch Service and Feedback, Izv. ANSSSR, Tekhnicheskaya Kibernetica 1 (1980), 112-113.

[4] Abolnikov, L.M. and Dukhovny, A.M., Necessary and Sufficient Conditions for the Ergodicity of Markov Chains with Transition $\Delta_{m, n}\left(\Delta_{m, n}^{\prime}\right)$ - Matrix, Journal of Applied Mathematics and Simulation 1 (1987): 13-24.

[5] Neuts, M.F., Queues Solvable Without Rouche's Theorem, Operations Research 27 No. 4 (1979): 767-781. 


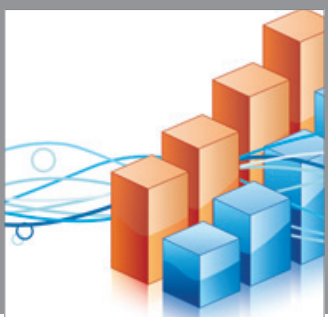

Advances in

Operations Research

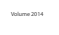

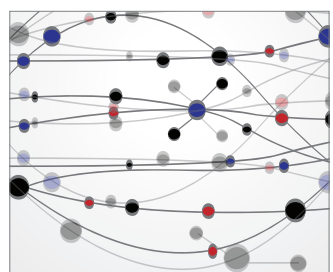

\section{The Scientific} World Journal
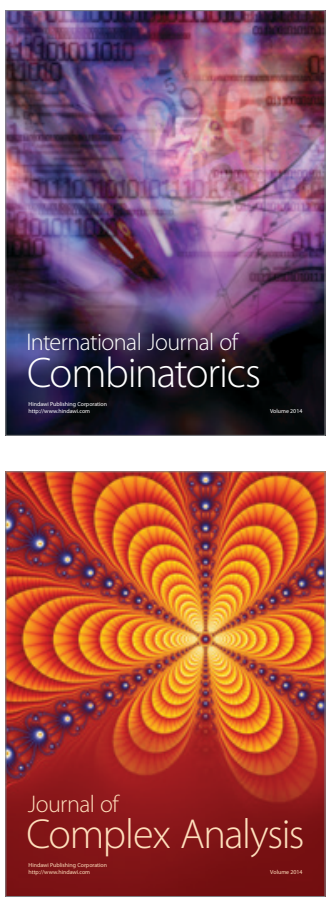

International Journal of

Mathematics and

Mathematical

Sciences
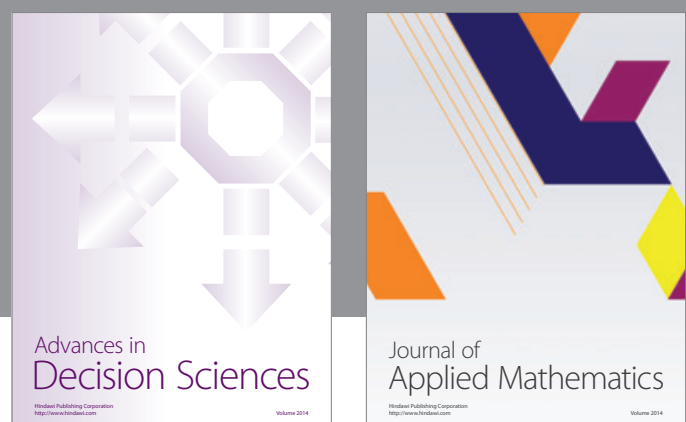

Journal of

Applied Mathematics
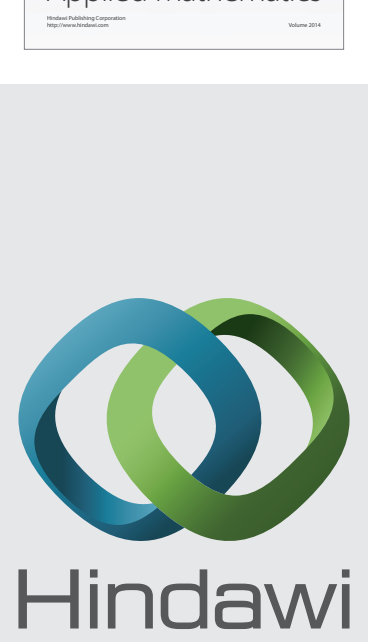

Submit your manuscripts at http://www.hindawi.com
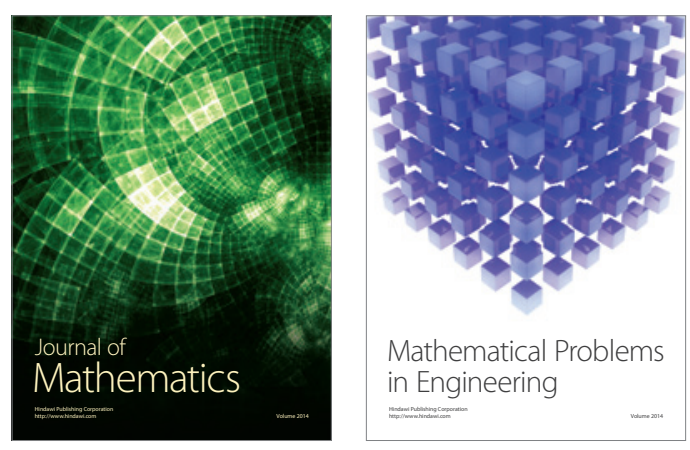

Mathematical Problems in Engineering
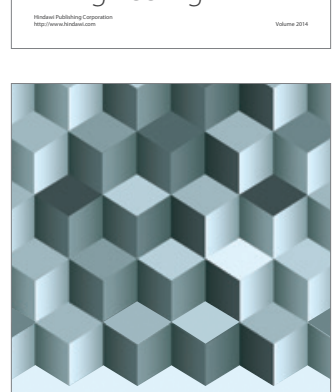

Journal of

Function Spaces
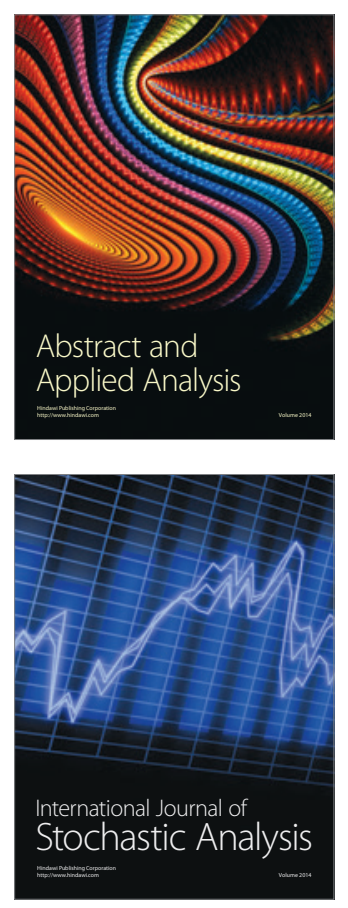

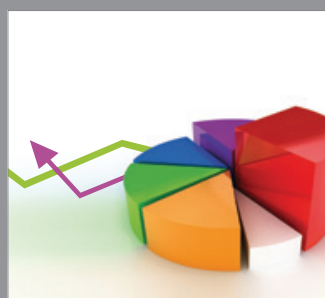

ournal of

Probability and Statistics

Promensencen
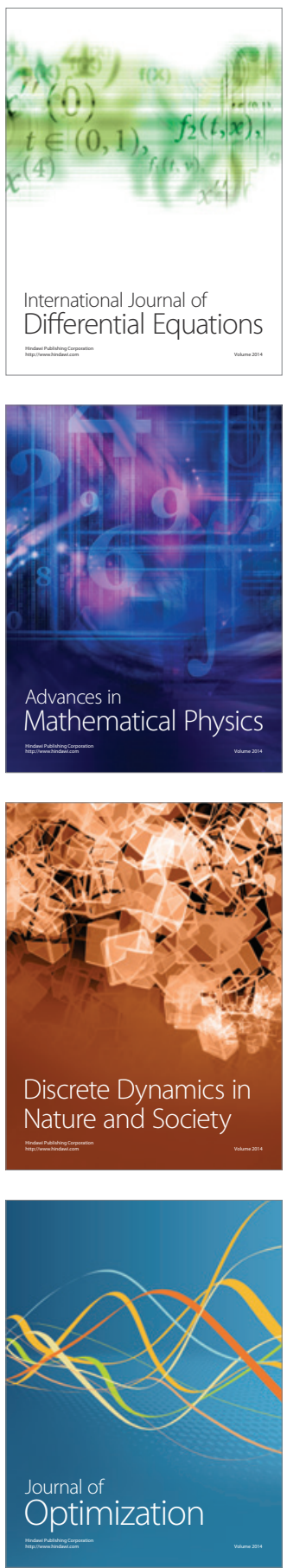